\title{
APPRAISAL OF TAKAGI-SUGENO TYPE NEURO-FUZZY NETWORK SYSTEM WITH A MODIFIED DIFFERENTIAL EVOLUTION METHOD TO PREDICT NONLINEAR WHEEL DYNAMICS CAUSED BY ROAD IRREGULARITIES
}

\author{
Hamid Taghavifar ${ }^{1}$, Asad Modarres Motlagh ${ }^{2}$, Aref Mardani ${ }^{3}$, Ali Hassanpour ${ }^{4}$, \\ Ashkan Haji Hosseinloo ${ }^{5}$, Leyla Taghavifar ${ }^{6}$, Chongfeng $\mathrm{Wei}^{7}$ \\ 1,2,3,4 Dept of Mechanical Engineering of Biosystems, Urmia University, Iran \\ ${ }^{5}$ Dept of Mechanical Engineering, Massachusetts Institute of Technology, United States \\ ${ }^{6}$ Dept of Electrical Engineering Department, Islamic Azad University, Iran \\ ${ }^{7}$ School of Mechanical Engineering, University of Birmingham, United Kingdom
}

Submitted 23 January 2015; resubmitted 9 July 2015, 2 October 2015, 16 December 2015; accepted 15 March 2016

\begin{abstract}
Wheel dynamics play a substantial role in traversing and controlling the vehicle, braking, ride comfort, steering, and maneuvering. The transient wheel dynamics are difficult to be ascertained in tire-obstacle contact condition. To this end, a single-wheel testing rig was utilized in a soil bin facility for provision of a controlled experimental medium. Differently manufactured obstacles (triangular and Gaussian shaped geometries) were employed at different obstacle heights, wheel loads, tire slippages and forward speeds to measure the forces induced at vertical and horizontal directions at tire-obstacle contact interface. A new Takagi-Sugeno type neuro-fuzzy network system with a modified Differential Evolution (DE) method was used to model wheel dynamics caused by road irregularities. DE is a robust optimization technique for complex and stochastic algorithms with ever expanding applications in real-world problems. It was revealed that the new proposed model can be served as a functional alternative to classical modeling tools for the prediction of nonlinear wheel dynamics.
\end{abstract}

Keywords: fuzzy system; wheel dynamics; obstacle; off-road; tire-obstacle contact; modeling.

\section{Introduction}

The forces between the road and the vehicle are transferred only through pneumatic tires. Being a substantial component of a vehicle, wheels are responsible for producing the forces for traversing and controlling the vehicle as well as those of braking, ride comfort, steering, maneuvering and following the road irregularities (Taghavifar, Mardani 2013b; Taghavifar 2015). Determination of the forces occurred during a driving wheel traversing over an obstacle and surface irregularity is a difficult task and a challenging problem due to nonlinear dynamics of the wheel and a complex tire-road interaction process.

The road irregularities would bring about variations in the compressive and extensive forces in both radial and lateral directions while the tire rotates about its axis. Tire forces are divided into three axes: radial, lateral, and tangential. The radial axis runs from the tire center toward the tread, and is the vertical axis running from the traversing path toward the vehicle that supports the vehicle weight. Concerned with the tire running on an uneven road, a varying force will be exerted into the vehicle which is the source of various ride disturbances. Generally speaking, during a traversing with no maneuvering, the two forces of radial and tangential are of greater importance. While the tangential force is responsible to the production of the required traction force for the vehicle to keep moving, obstacle traversing affects both of the forces. The qualitative and quantitative insight into the horizontal and vertical forces induced at tire-obstacle interface is substantial requisites of vehicle designers and manufacturers due to many reasons such as controlling vehicle, overturning, ride comfort at off-road grounds, vehicle axle damages, and power reduction at tire-obstacle contact time. The vehicle responses during off-road operation are dependent on the road conditions and vehicle parameters such as vehicle speed, vehicle weight, and tire slippage. 
A survey was conducted to appraise the impact of tire characteristics on variations of wheel load and vibrations transmitted from the ground to the tractor rear axle. Vertical wheel load of the left and right rear wheels were quantified by means of strain-gage-based transducers (Nguyen, Inaba 2011). The time histories and frequency compositions of synthesized data indicated that traversing irregularities excited the wheel load at their natural frequencies and harmonics (Nguyen, Inaba 2011). A mathematical modeling attempt was undertaken for vehicle traversing over an obstacle and proposed an analytical tool for determining the obstacle height that the wheel can overcome the instability (Thomas, Vantsevich 2010). Collision of automobile wheels with a vertical obstacle was performed and the required force and the minimal speed of driving for disassembling of the tire from the wheel rim after the collision with an obstacle were determined (Sokolovskij et al. 2007). It was also reported that the values of this force and speed depends upon the height of the obstacle and the angle of collision with the obstacle. Analytical and experimental attempts for an off-road vehicle ride dynamics model was also performed while the random roughness properties of the two parallel tracks of terrain were analyzed in view of equivalent undeformable terrain (Pazooki et al. 2012). Open literature exists with some other studies given the attention to the vehicle dynamics of the wheels traversing over uneven surfaces (Senatore, Sandu 2011; Mason et al. 2012) and vehicle overturn due to movement steep slope/obstacle as an indication of road irregularities (Ahmadi 2011; Gravalos et al. 2011).

As previously mentioned, the importance of ascertaining the horizontal and vertical forces exerted to the tire while traversing over obstacles is of priority given that vehicle dynamics is synthesized with a detailed insight into the mechanics of wheel-obstacle contact. This investigation explores the nonlinear wheel dynamics caused by road irregularities as affected wheel load, tire slippage, speed, obstacle height, and obstacle geometry with the appraisal of Takagi-Sugeno type neuro-fuzzy network system coupled to a modified Differential Evolution (DE) method. This paper is organized as follows. In Section 1 a brief explanation about experimental data collection is given. In Section 2, we introduce the proposed algorithm (i.e. Takagi-Sugeno type neuro-fuzzy network with modified DE System). Section 3 discusses the obtained results and presents the results from the experiments and modeling processes. Last section concludes the paper.

\section{Experimental Data Collection}

The provision of controlled testing environment is crucially significant for the reliability of the results and the outcomes of the study. Hence, a Single-Wheel Tester (SWT) inside a soil bin facility manufactured in the Department of Mechanical Engineering of Biosystems of Urmia University, Iran, was used to conduct the required experiments. The soil bin channel with the $24 \mathrm{~m}$ length, $2 \mathrm{~m}$ width and $1 \mathrm{~m}$ depth was filled with the soil texture of test region. The holistic system is consisted of soil bin channel, SWT and the carriage. The SWT was connected to the carriage to be enabled to traverse during the soil bin. The carriage was powered with a $22 \mathrm{~kW}$ electromotor, which was in turn, connected to the inverter to manage the start/stop and velocity control procedures. The power transmission was carried out through the electromotor to the chain system that was linked to the carriage. The carriage was traversing in the channel by means of four ball bearings positioned on the sidewalls of the soil bin. The SWT was connected to the carriage through an L-shaped part and also four horizontal arms each accommodating S-shaped Bongshin load cell with $500 \mathrm{~kg}$ capacity. It is worth to note that the horizontal load cells were used to measure the horizontal forces applied to the wheel. A U-shaped frame was used as housing to the tire and a three-phase electromotor of $5 \mathrm{~kW}$ to power the driving wheel. An appropriate inverter was also used to control the rotational velocity delivered to the wheel shaft and therefore; the linear velocity was adjustable. It is worth mentioning that the linear speed difference between the carriage and the SWT yielded different levels of adjustable slippage. Furthermore, the SWT was connected to the L-shaped frame by a power bolt rod (to adjust the applied wheel load) which was connected to a vertically situated Sshaped load cell, which was responsible to measure the load variations while traversing over the obstacle and irregularities. The load cells were connected to Bongshin digital indicators, which were in turn in connection with a data logger with RS232 output signals. The data were subsequently sent to the laptop computer to be stored and processed with the frequency of $30 \mathrm{~Hz}$. The general soil bin facility along with the SWT is shown in Fig. 1.

For all the experiments the tire inflation pressure was maintained at $131 \mathrm{kPa}$. Two shapes of triangular and Gaussian obstacles were used in the study each at three heights of 2, 3 and $4 \mathrm{~cm}$ while two wheel loads of 1 and $2 \mathrm{kN}$ were considered. Furthermore, two levels of slippage were induced at 10 and $20 \%$. Three forward velocities for the carriage were planned at three levels of $1.08,1.8$ and $2.52 \mathrm{~m} / \mathrm{s}$. In order to remove the soil effect

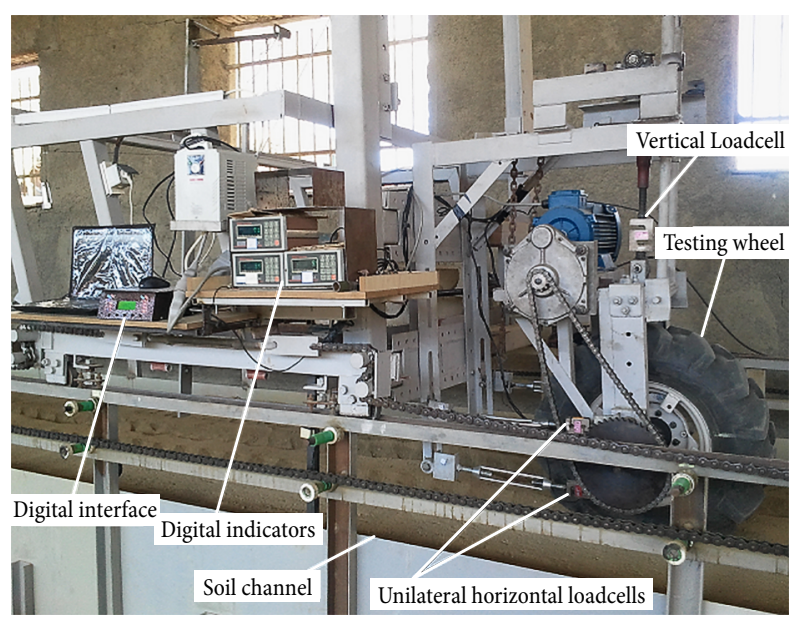

Fig. 1. Soil bin testing facility and the components 


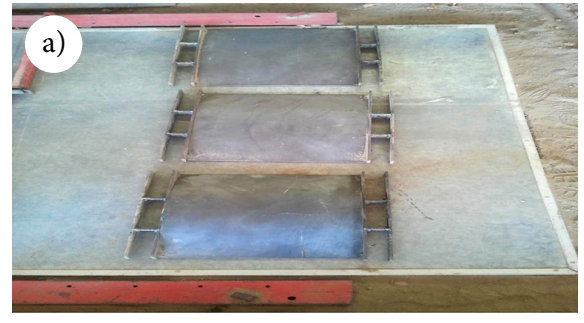

Semicircular obstructions at different heights

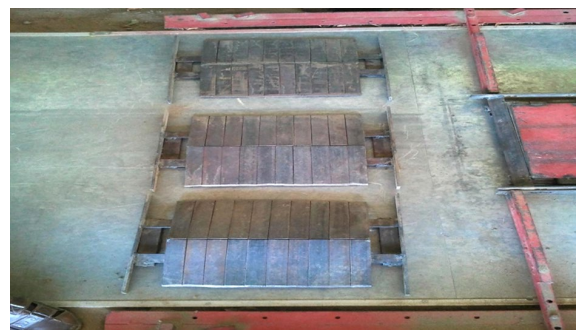

Triangular obstructions at different heights
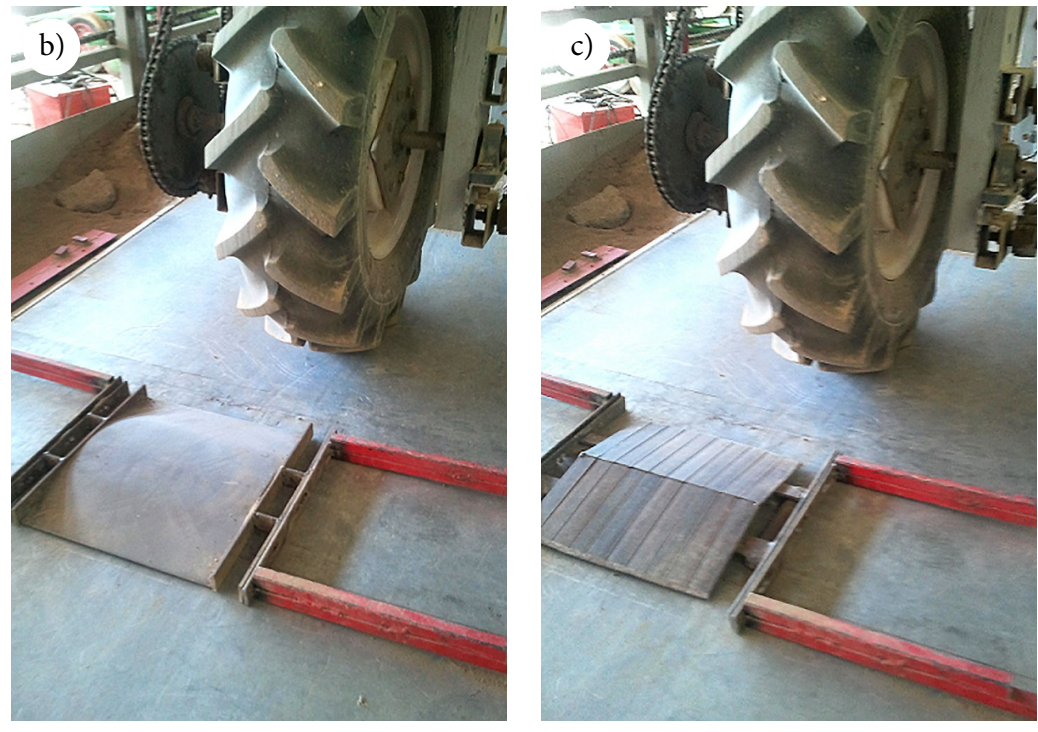

Fig. 2. An overview on the obstacles used in the experiments (a), the tester wheel traversing Gaussian obstacle (b) and the tester wheel traversing triangular obstacle (c)

on the experiment outputs due to the soil nonhomogeneous properties, a wooden board with $2 \mathrm{~m}$ width and $3 \mathrm{~m}$ length was used to mount the obstacles on. The tests were carried out in a randomized complete block design with three replicates. The obstacles situated in the traversing direction of the wheel are depicted in Fig. 2.

When a vehicle traverses road irregularities or obstacles, it may experience highly dynamic loading, and the different subsystems respond with varying degrees of nonlinearity. It is noteworthy that the nonlinearity of wheel dynamics lies in the random shocks and kinetics of motion in longitudinal and normal directions. The impact force variations is a representative of nonlinear dynamic in time domain that can be attenuated based on wheel running condition. The longitudinal variations will be demonstrated through the tractive parameters such as net traction, rolling resistance, braking, etc. and wheel load variations, tire deflection are representatives of the variations in vertical direction. There are several important parameters, which govern tire-ground interaction at longitudinal and normal direction such as wheel load, road profile and forward speed as well as their interactions. Tire slip region is an additional factor to the complexity of the system. The effect of all influential factors in the nonlinear dynamics of wheel-ground interaction will be explicitly achieved in the experimental results. The elastic tire rolling on ground to investigate some operating variables such as tire normal force, inflation pressure and slippage that determine tire contact patch longitudinal and vertical forces is the major factor subject that affects the dynamics of vehicle.

Wheel as the unique linkage between the vehicle and ground is subject to all the forces and torques acting on the vehicle. Of the most important vehicle characteristics that can drastically affect the vehicle performance is its weight. Wheel load represents the load that each of wheels support from the total weight of vehicle. Dur- ing the experiments, the wheel load can be adjusted to investigate the effect of total vehicle weight on its kinetics. Tire slippage, also known as travel reduction, is the relative motion between a tire and the ground and can be produced by the tire's rotational speed being greater or less than the free-rolling speed.

This study is free from strict theoretical formulation and attempts to solve the problem by adoption of a promising tool of artificial intelligence (i.e. Adaptive Neuro-Fuzzy Inference System - ANFIS) that has shown an outstanding fit to deal with nonlinear problems. Hence, instead of mathematical modelling, the experimental data will be fed to the modeling tool and the results of the model will be compared and verified by the actual data to determine the modeling accuracy.

\section{Takagi-Sugeno Type Neuro-Fuzzy Network with Modified Differential Evolution System}

As a stochastic function minimizer, $\mathrm{DE}$ is a method that optimizes a problem by iteratively trying to improve a candidate solution with regard to a given measure of quality (Fleetwood 2004). DE is used for multidimensional real-value functions with no use of the gradient of the problem. DE optimizes a problem by keeping a population of candidate solutions and creating new candidate solutions by combining existing ones according to its simple formulae, and then keeping whichever candidate solution has the best score or fitness on the optimization problem at hand. In this way, the optimization problem is treated as a black box that merely provides a measure of quality given a candidate solution. The DE calculations are described as following (Fleetwood 2004).

For a given function to be optimized the following problem function is described $f: X \subseteq R^{D} \rightarrow R$ at the region $X \neq \phi$, the minimization problem is defined as $x^{*} \in X$ hence $f\left(x^{*}\right) \leq f(x) \forall x \in X$, where $f\left(x^{*}\right) \neq-\infty$. 
For an objective function of $D$ real parameters, the size of the population $N$ is ascertained. The parameter vectors are therefore yielded as:

$$
\begin{aligned}
& x_{i, G}=\left[x_{1, i, G}, x_{2, i, G}, \ldots x_{D, i, G}\right] \\
& \text { for } i=1,2, \ldots, N,
\end{aligned}
$$

where: $G$ is the number of generation.

The higher and lower limits for each parameter are described as:

$$
x_{j}^{L} \leq x_{j, i, 1} \leq x_{j}^{U},
$$

where the randomly selected initial parameters were uniformly on the intervals $\left[x_{j}^{L}, x_{j}^{U}\right]$.

After the initialization step is finished, the parameter vectors goes through mutation, recombination and selection stages. In the mutation the search space is expanded.

For a given parameter vector $x_{i, G}$ randomly select three vectors $x_{r 1, G}, x_{r 2, G}$ and $x_{r 3, G}$ such that the indices $i, r_{1}, r_{2}$ and $r_{3}$ are distinct. The weighted difference of two of the vectors to the third are subsequently added:

$$
v_{i, G+1}=x_{r 1, G}+F\left(x_{r 2, G}-x_{r 3, G}\right),
$$

where: $v_{i, G+1}$ is the donor vector. The mutation factor $F$ is a constant from $[0,2]$.

Recombination incorporates successful solutions from the previous generation:

$$
u_{j, i, G+1}=\left\{\begin{array}{ll}
v_{j, i, G+1} & \text { if } \text { rand }_{j, i} \leq C R \text { or } j=I_{\text {random }} \\
x_{j, i, G} & \text { if } \text { rand }_{j, i} \leq C R \text { and } j \neq I_{\text {random }}
\end{array}\right\},
$$

where: rand $_{j, i} \sim U[0,1] ; I_{\text {random }}$ is a random integer from $[1,2, \ldots, D]$.

In selection step, the target vector $x_{i, G}$ is compared with the trial vector $v_{i, G+1}$ and the one with the lowest function value is presented to the next generation:

$$
\begin{aligned}
& x_{i, G+1}=\left\{\begin{array}{ll}
u_{i, G+1} & \text { if } f\left(u_{i, G+1}\right) \leq f\left(x_{i, G}\right) \\
x_{i, G} & \text { otherwise }
\end{array}\right\}, \\
& i=1,2, \ldots, N .
\end{aligned}
$$

Mutation, recombination and selection iterate until at least one criterion is reached.

Takagi-Sugeno neuro-fuzzy computing method is the combination of neural network theorem and Mamdani-type fuzzy logic system technique as a robust tool for solving various problems with high level of uncertainty in science and engineering problems concerned with issues such as pattern recognition, identification, controlling.

The first-order Sugeno fuzzy model two fuzzy 'IfThen' rules can be expressed as:

$$
\begin{aligned}
& \text { If } x=A_{1} \text { and } y=B_{1} \text { then } f_{1}=p_{1} x+q_{1} y+t_{1} ; \\
& \text { If } x=A_{2} \text { and } y=B_{2} \text { then } f_{2}=p_{2} x+q_{2} y+t_{2} .
\end{aligned}
$$

Generally speaking, there are layers in the structure of Takagi-Sugeno fuzzy computation method. The first layer consists of input variable membership functions (the fuzzification in which each node represents a membership function) where the nodes are equal to the number of input variables with node functions. A schematic Takagi-Sugeno fuzzy system architecture is depicted in Fig. 3.

$$
\begin{aligned}
& O_{i}^{1}=\mu_{A i}(x), i=1,2 ; \\
& O_{i}^{1}=\mu_{B-i}(y), i=3,4,
\end{aligned}
$$

where: $\mu_{A}(x), \mu_{B}(y)$ are membership functions with different forms such as Gaussian, bell-shaped, triangular, trapezoidal, etc.

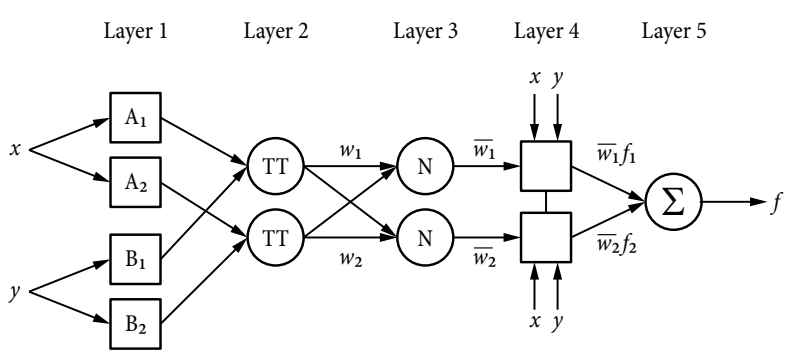

Fig. 3. A schematic Takagi-Sugeno fuzzy system architecture

The second layer (so called membership layer) yields the strength of the rules by means of multiplication operator in each node. It takes the input values from the previous layer and operates as a membership function to characterize the fuzzy sets of the corresponding input variables:

$$
O_{i}^{2}=\mu_{A i}(x) \mu_{A i}(y), i=1,2 .
$$

Third layer as the normalization layer (or the rule layer), normalizes the firing strength of the rules. Each node in these layers computes the weights, which are normalized. In this layer, the ratio of the firing strength of a rule to the sum of the total is calculated as following:

$$
O_{i}^{3}=\frac{w_{i}}{w_{1}+w_{2}}, i=1,2 .
$$

The fourth layer is defuzzification layer, which represents the output values obtained from the inference of rules. Furthermore, this layer includes adaptive nodes that calculate a linear function with coefficients that are adapted by using the error function of the feed-forward neural network (Karaağaç et al. 2012):

$$
\bar{w}_{i} f_{i}=\bar{w}_{i}\left(p_{i} x+q_{i} y+t_{i}\right) .
$$

The last layer, which has a single node refers to the summation of the inputs of the nodes from previous layer. The output $\mathrm{f}$ is calculated as following:

$$
f=\bar{w}_{1} f_{1}+\bar{w}_{2} f_{2}=\frac{w_{1} f_{1}+w_{2} f_{2}}{w_{1}+w_{2}} .
$$

A typical Takagi-Sugeno fuzzy computing approach adopts hybrid method of the gradient descent and the least-squares method methodology to define the optimal specifications for tuning the membership 
functions based on the back-propagation technique that computes error signals repetitively from the output layer backward to the input nodes. However, we used a hybrid method of Takagi-Sugeno type neuro-fuzzy network system with a modified DE optimization method.

For any developed model, a necessary step is to assess the model on account of some performance criteria. This step ensures that the developed model has enough capacity for implementations. Hence, two statistical metrics of Root Mean Squared Error (RMSE), and coefficient of determination $\left(R^{2}\right)$ were applied as following:

$$
\begin{aligned}
& R M S E=\sqrt{\frac{\sum_{i=1}^{n}\left(Y_{\text {predicted }}-Y_{\text {actual }}\right)^{2}}{n}} ; \\
& R^{2}=\frac{\sum_{i=1}^{n}\left(Y_{\text {predicted }}-Y_{\text {mean }}\right)^{2}}{\sum_{i=1}^{n}\left(Y_{\text {actual }}-Y_{\text {mean }}\right)^{2}},
\end{aligned}
$$

where: $Y_{\text {actual }}, Y_{\text {predicted }}$ are measured and predicted values by the developed models.

\section{Results and Discussion}

It is well-recognized that wheel dynamics is of great complexity and nonlinearity to be assessed as a prototype of the measured vertical and horizontal induced forces during tire-triangular shaped obstacle contact is demonstrated in Fig. 4 concerned with the wheel load of $2 \mathrm{kN}$, slippage of $10 \%$ and obstacle height of $2 \mathrm{~cm}$, forward speed of $1.08 \mathrm{~m} / \mathrm{s}$. As appreciated from Fig. 4, the created vertical force is greater than that of horizontal force. Furthermore, the vertical force is greatly affected by the impact force with greater range of amplitude variation (compressive/extensive loads). The load variations are very complex being pivotal on the natural frequency of the system as well as the system response to the surface condition. The difference between the vertical and horizontal induced forces are attributable to the impact point position, loading condition, etc. In the interest of a balanced vehicle traversing, the accuracy and reliability of the complete vehicle model has to produce rational relation to the performance of the applied tire model. Considering the fully nonlinear and tire dynamic, the developed models are very complex (Lugner et al. 2005), thus; the models should deal with the stochastic vehicle vibrations happening traversing on rough road that entail strength-relevant component loads (Rill 2006).

Based on the extension of the experiments, 108 tests were conducted resulting to 108 data samples for the vertical induced force and 108 data samples for the horizontal induced force. The total data were shuffled into two sets of training and testing with equal portions. The developed network had three membership functions for input fuzzification. At the beginning, the developed system was trained with the training input followed by testing phase to determine the vertical and horizontal a)

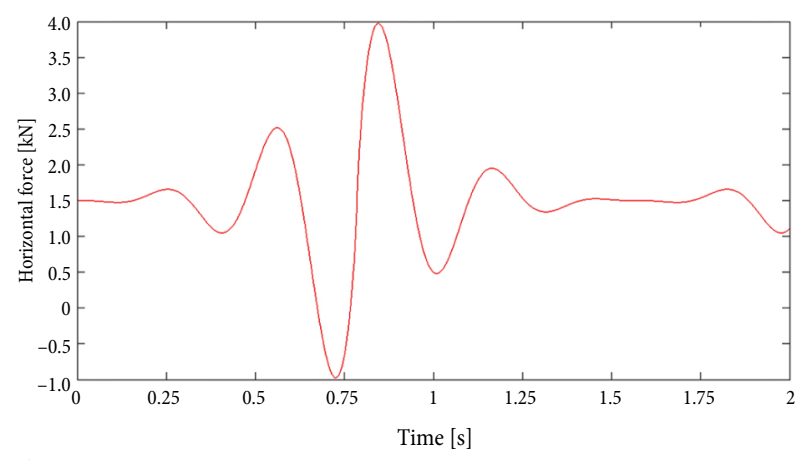

b)

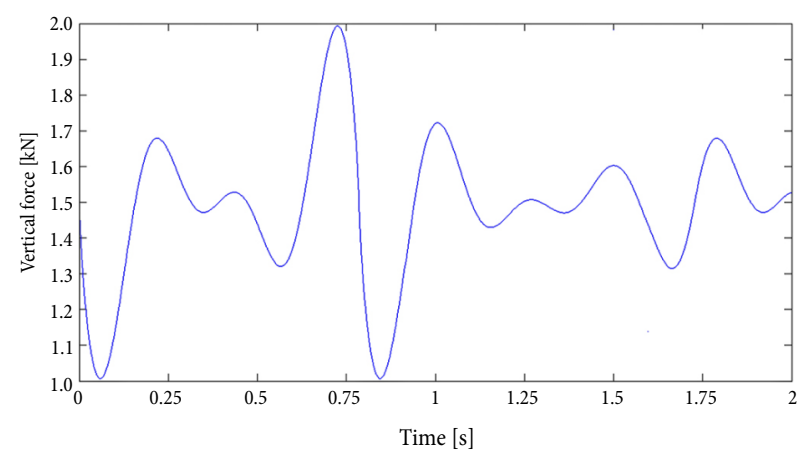

Fig. 4. A prototype of the measured horizontal (a) and vertical (b) induced forces during tire-triangular shaped obstacle contact at wheel load of $2 \mathrm{kN}$, slippage of $10 \%$, obstacle height of $2 \mathrm{~cm}$, and forward speed of $1.08 \mathrm{~m} / \mathrm{s}$

forces. Since straight-line functions were adopted due to theirs considerable simplicity where the uniformly triangular membership functions were selected owing to superior accuracy (Taghavifar, Mardani 2014). In this manner trapezoidal and triangular membership functions were employed. The belonging of a factor to a fuzzy set is accompanied with membership functions. A membership function is a curve defines how every point in the input is mapped to a membership value between 0 and 1 . The input space is sometimes referred to the universe of discourse, a visualized name for a simple notion. In order to verify the merit of the new constructed model, the classical ANFIS was synthesized and the obtained results are tabulated in Table. As appreciated from Table, it is obvious that Takagi-Sugeno Fuzzy model with DE optimization method (TSF-DE) produces more reliable solutions to the nonlinear wheel dynamics problem for both vertical and horizontal forces when compared to the classical ANFIS models. The statistical metrics in Table further confirms that the Takagi-Sugeno type system modified by DE model has ability to be utilized for solving the nonlinear vehicle dynamics with favorable level of reliability and preciseness. Fig. 5 and 6 depict Takagi-Sugeno type system modified by $\mathrm{DE}$ decision surface for the estimation of horizontal and vertical induced forces of the driving wheel at tire-obstacle interface, respectively. These surfaces are nonlinear and monolithic surfaces and represent fuzzy decision surfaces for given inputs (Petković et al. 2015). 
Table. The characteristics of the best structure of developed models

\begin{tabular}{|c|c|c|c|c|c|}
\hline Item & Type of MF & RMSE for VF & $R^{2}$ for VF & RMSE for HF & $R^{2}$ for HF \\
\hline ANFIS & Trapezoidal & 0.1587 & 0.94 & 1.0229 & 0.93 \\
\hline TSF-DE & Trapezoidal & 0.1291 & 0.98 & 1.2313 & 0.94 \\
\hline ANFIS & Triangular & 0.0952 & 0.95 & 0.9857 & 0.94 \\
\hline TSF-DE & Triangular & $\mathbf{0 . 0 6 9 1}$ & $\mathbf{0 . 9 7}$ & $\mathbf{0 . 3 1 0 0}$ & $\mathbf{0 . 9 6}$ \\
\hline
\end{tabular}

Notes: MF - Membership Function; VF - Vertical Force; HF - Horizontal Force; TSF-DE - Takagi-Sugeno Fuzzy model with DE optimization; the boldfaced values show the outperforming models.

a)

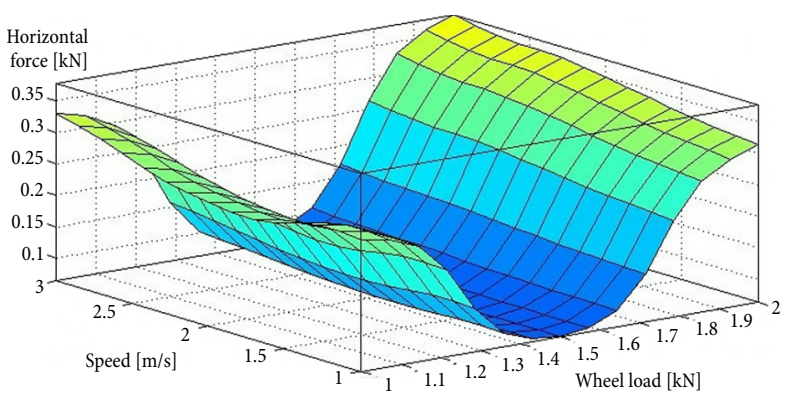

c)

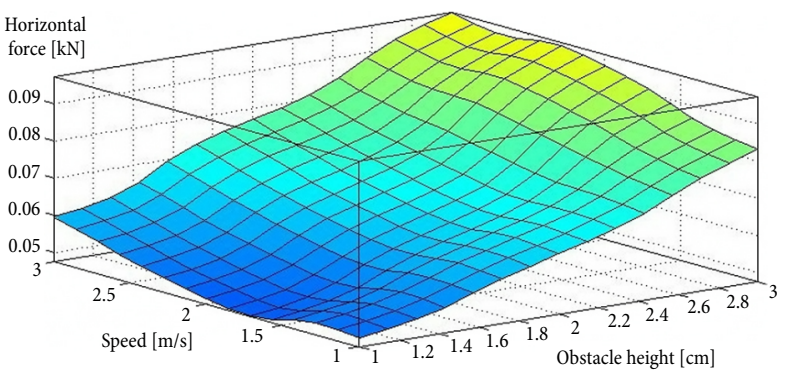

e)

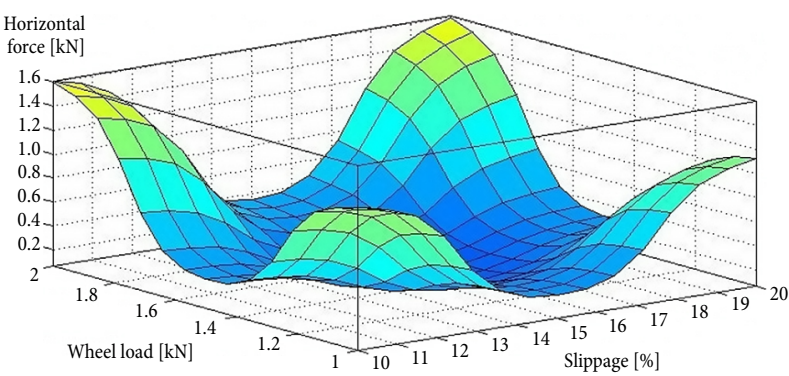

b)

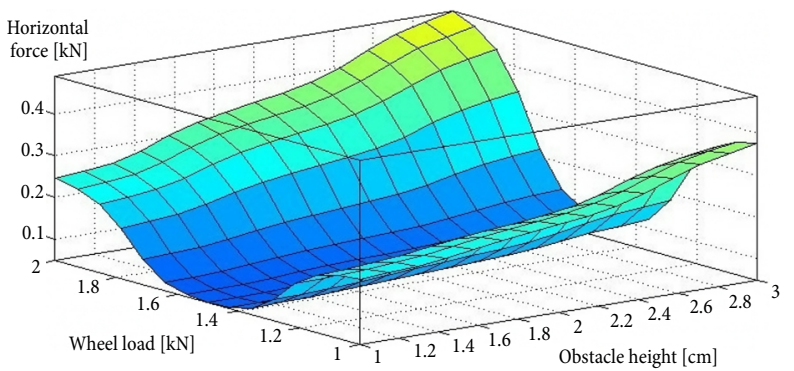

d)

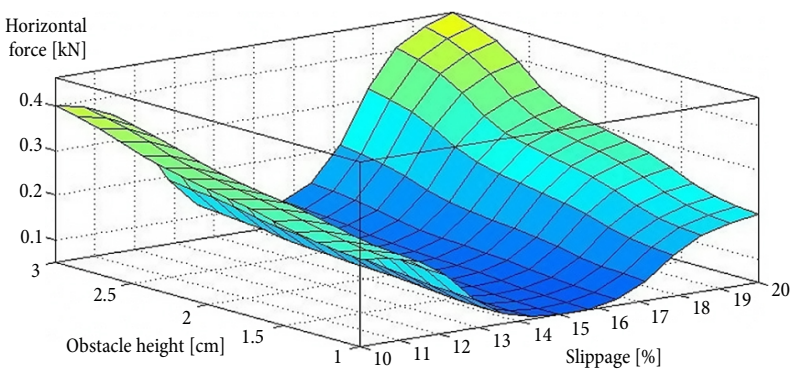

f)

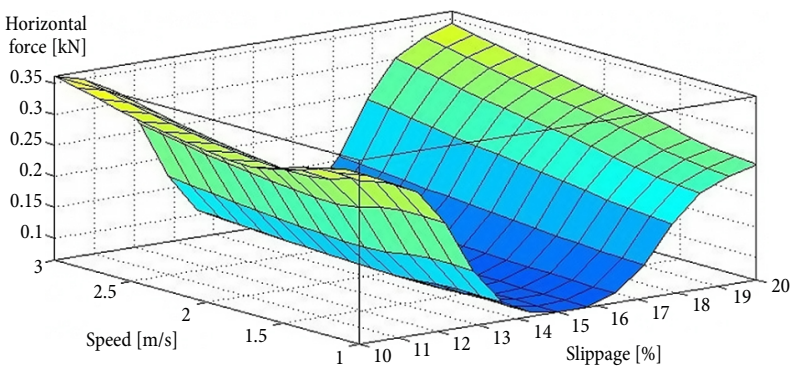

Fig. 5. The decision surfaces of the induced horizontal force with respect to the different inputs of: $a$ - speed vs. wheel load; $b$ - obstacle height vs. speed; c - obstacle height vs. wheel load; $d$ - slippage vs. obstacle height; e - slippage vs. wheel load; $f$ - slippage vs. speed

The depicted decision surfaces can contribute to the knowledge of an expert concerned with the influence of the input variables on the output signal. At the outperforming Takagi-Sugeno type system modified by DE structure, the variations of error for each data sample are presented as well as error histogram in the training and testing phases. A comparison between Figs. 7a and $7 \mathrm{~b}$ as well as those of Fig $8 \mathrm{a}$ and $8 \mathrm{~b}$ indicates that the dispersion degree of training phase is slighter when compared to the testing phase. It should be divulged that the proposed Takagi-Sugeno type system modified by DE model can be embedded as a module with computa- tionally efficiency and adaptability. Neuro-fuzzy system has shown a reasonable applicability for longitudinal control of autonomous vehicles putting this method as a computationally efficient approach to solve vehicle dynamics related fields (Pérez et al. 2010). A comparison was made between a non-fuzzy approach and ANFIS model using a very well documented study with finite element method approach. Based on Cho et al. (2005), the longitudinal and vertical error for this study are about 9.3 and 7\%, respectively. The error for TSF-DE model of the present study for longitudinal and horizontal are 0.0691 and $0.31 \%$ (Table 1 ) and Fig. 7 and 8 . This 
a)

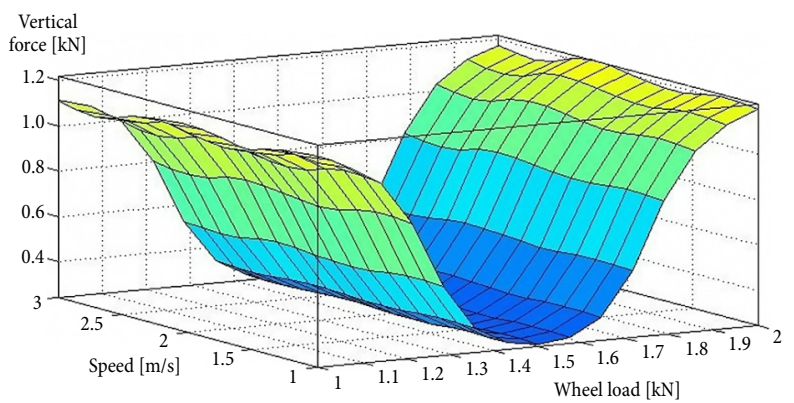

c)

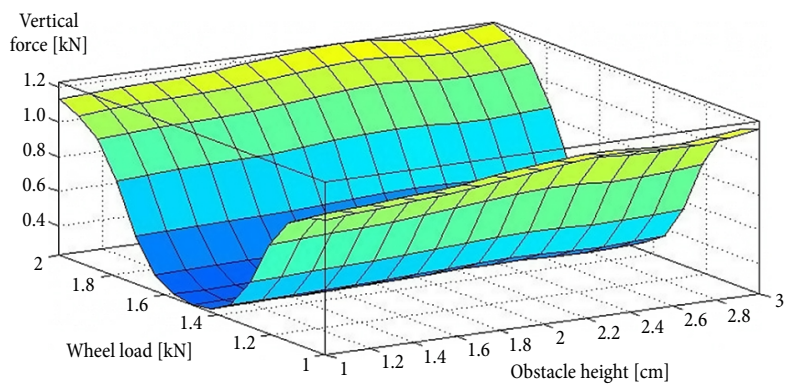

e)

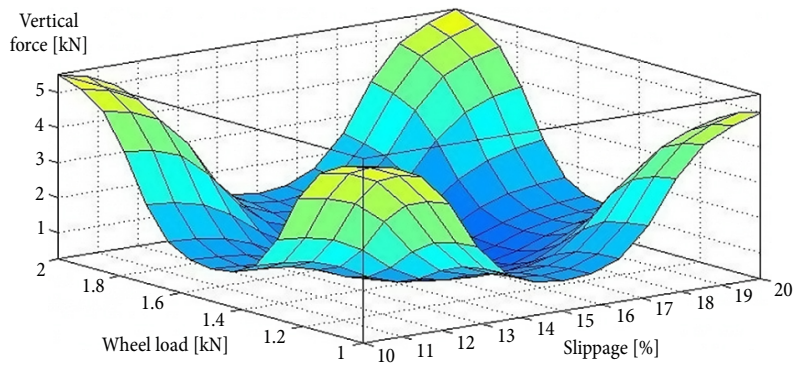

b)

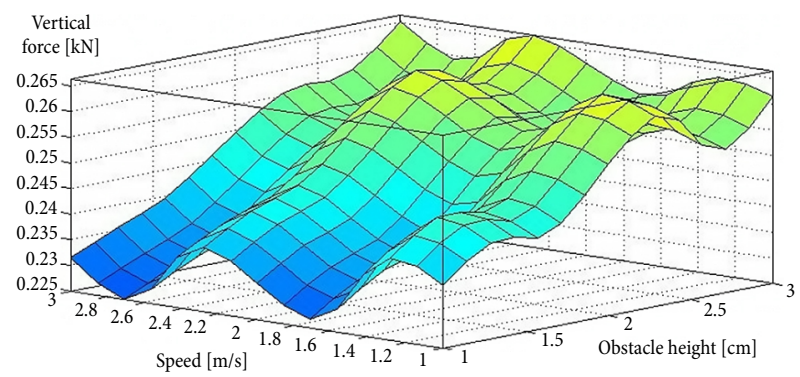

d)

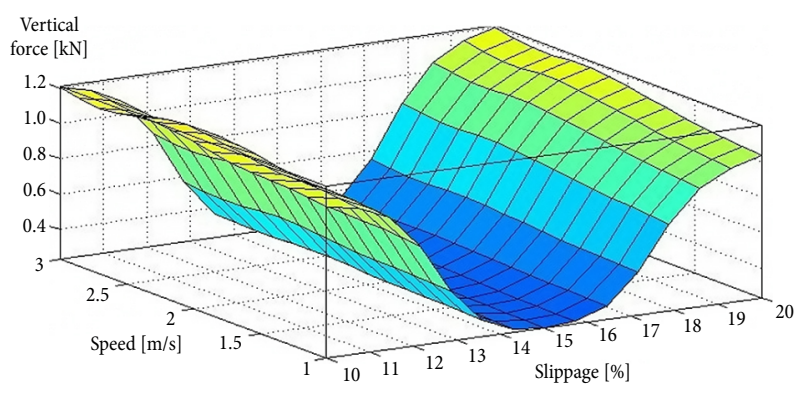

f)

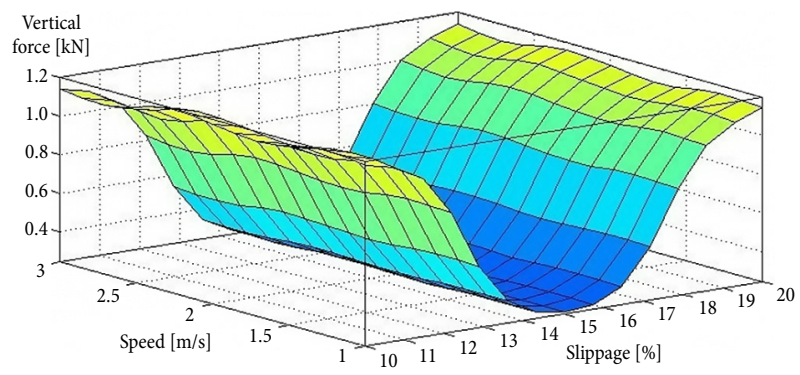

Fig. 6 . The decision surfaces of the induced vertical force with respect to the different inputs of: $a$ - speed vs. wheel load; $b$ - obstacle height vs. speed; $c$ - obstacle height vs. wheel load; $d$ - slippage vs. obstacle height; e - slippage vs. wheel load; $f$ - slippage vs. speed

shows the promising ability of using the new model used in the present study. A rule-based Mamdani max-min fuzzy expert system for prediction of coefficient of motion resistance, as an important wheel kinetic product, that showed mean relative error about 10\% (Taghavifar, Mardani 2013a). The suitability and applicability of the proposed model is further confirmed when compared by the studies using classical fuzzy logic based models to predict different vehicle dynamic products (Taghavifar, Mardani 2013a, 2014, 2016). This is a further confirmation of the promising ability of TSF-DE to deal with the wheel-obstacle collision dynamics.

\section{Conclusions}

This study was aimed at prediction of wheel dynamics caused by road irregularities using Takagi-Sugeno type neuro-fuzzy network system with a modified DE method. The experimental phase to validate the developed model was implemented using a single-wheel testing rig in a soil bin facility that for provided a controlled experimental medium while different operating condition and tire parameters were included. Since straightline functions were adopted due to theirs considerable simplicity where the uniformly triangular membership functions were selected owing to superior accuracy. Based on the obtained results, TSF-DE yielded a model with RMSE of vertical force and horizontal force at 0.0691 , and 0.3100 , respectively. Coefficient of determination values of 0.97 and 0.96 were also obtained for vertical and horizontal forces, respectively. The outcome of this study indicated that TSF-DE is a promising tool for the prediction of different phenomena of wheel-ground kinetics and kinematics. It can also be used as an approach to yield a model that can be used in any future research framework without any need to repeat the experiments to find the behavior of wheel when collided by obstacles and cleats. Based on the obtained results, it is concluded that the TSF-DE further outperformed ANFIS to solve the problem at different membership function shapes. It was finally noted that the proposed model is applicable and suitable to be used for the modeling of wheel-obstacle collision with satisfactory performance when compared with numerical based simulation tools such as finite element method.

It is recommended to further investigate the proposed model particularly for the other vehicle dynamics situation such as vehicle stability, ride comfort and handling, maneuvering, etc. 

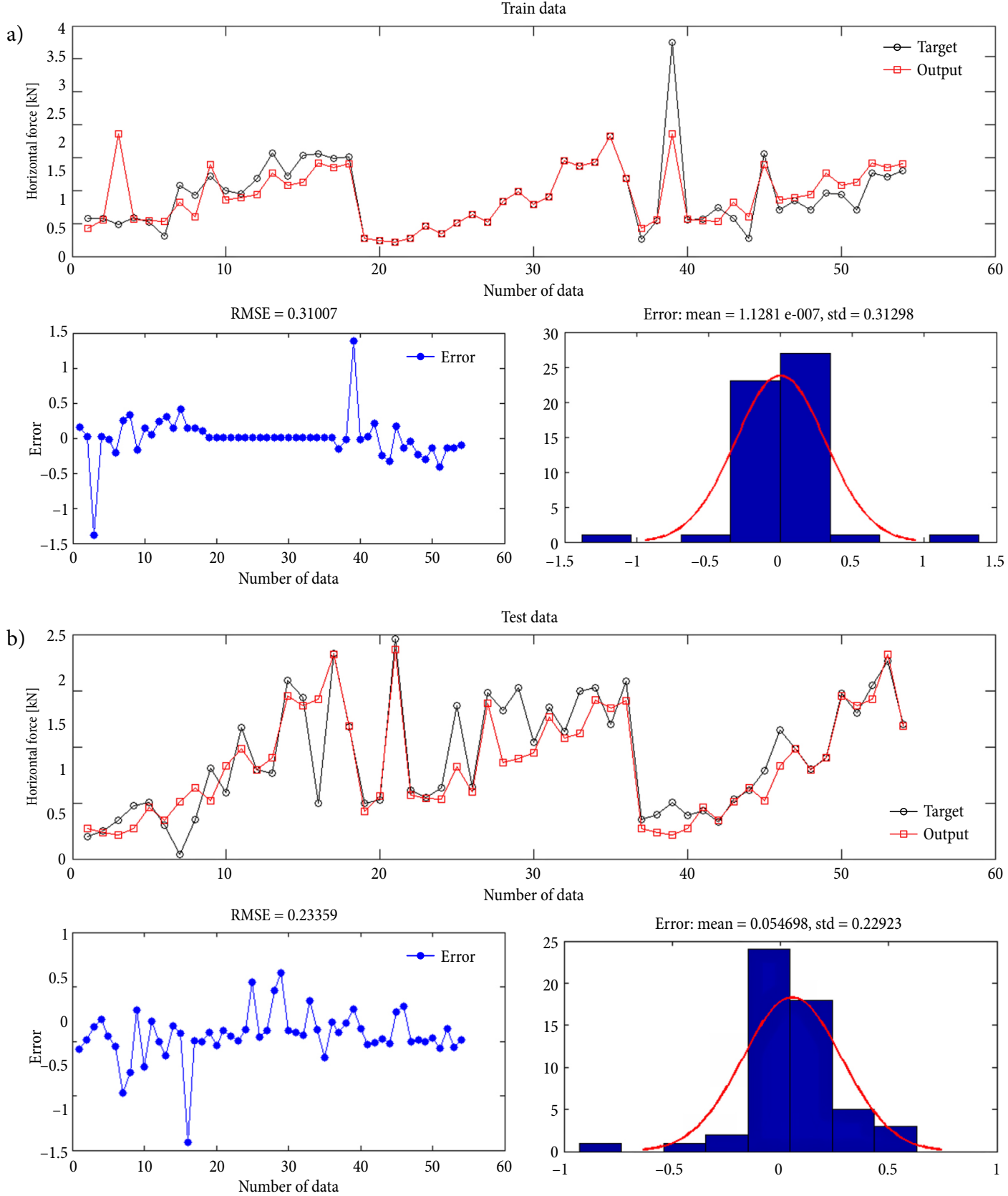

Fig. 7. The performance of the selected Takagi-Sugeno type system modified by DE model for the induced horizontal force with data mapping between the modeled and experimental values, RMSE during the number of data and error histogram for training (a) and testing (b) phases

\section{References}

Ahmadi, I. 2011. Dynamics of tractor lateral overturn on slopes under the influence of position disturbances (model development), Journal of Terramechanics 48(5): 339-346. http://doi.org/10.1016/j.jterra.2011.07.001

Cho, J. R.; Kim, K. W.; Jeon, D. H.; Yoo, W. S. 2005. Transient dynamic response analysis of 3-D patterned tire rolling over cleat, European Journal of Mechanics - A/Solids 24(3): 519-531. http://doi.org/10.1016/j.euromechsol.2005.01.004

Fleetwood, K. 2004. An introduction to differential evolution, in One-day Symposium: Multi-Agent Systems and Machine Learning, 26 November 2004, University of Queensland, Australia, 40 p. Available from Internet: http://www.maths. uq.edu.au/MASCOS/Multi-Agent04/Fleetwood.pdf
Gravalos, I.; Gialamas, T.; Loutridis, S.; Moshou, D.; Kateris, D.; Xyradakis, P.; Tsiropoulos, Z. 2011. An experimental study on the impact of the rear track width on the stability of agricultural tractors using a test bench, Journal of Terramechanics 48(4): 319-323.

http://doi.org/10.1016/j.jterra.2011.04.003

Karaağaç, B.; İnal, M.; Deniz, V. 2012. Predicting optimum cure time of rubber compounds by means of ANFIS, $\mathrm{Ma}$ terials \& Design 35: 833-838.

http://doi.org/10.1016/j.matdes.2011.03.062

Lugner, P.; Pacejka, H.; Plöchl, M. 2005 Recent advances in tyre models and testing procedures, Vehicle System Dynamics 43(6-7): 413-426.

http://doi.org/10.1080/00423110500158858 

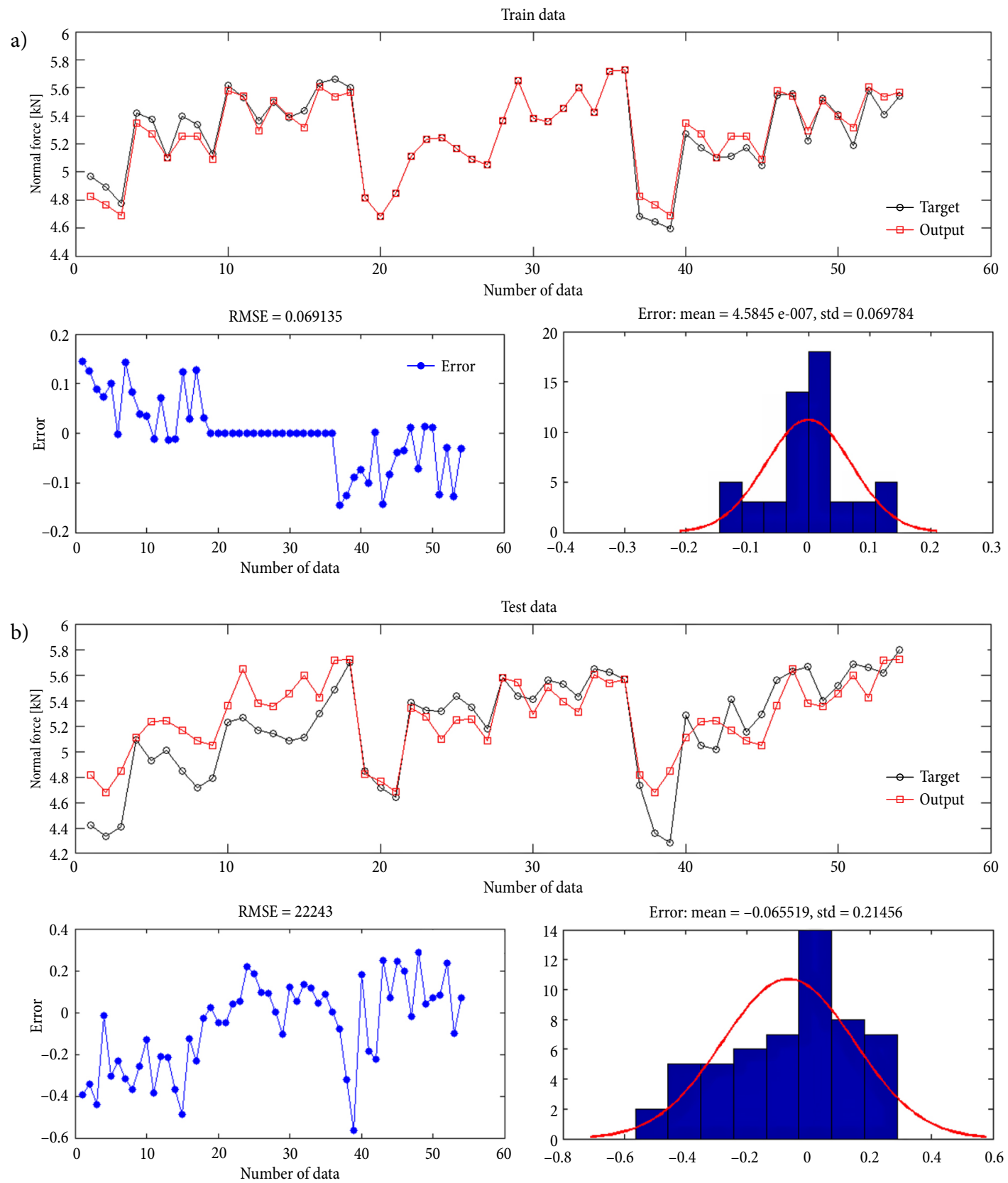

Fig. 8. The performance of the selected Takagi-Sugeno type system modified by DE model for the induced vertical force with data mapping between the modeled and experimental values, RMSE during the number of data and error histogram for training (a) and testing (b) phases

Mason, G. L.; Gates, B. Q.; Moore, V. D. 2012. Determining forces required to override obstacles for ground vehicles, Journal of Terramechanics 49(3-4): 191-196. http://doi.org/10.1016/j.jterra.2012.04.001

Nguyen, V. N.; Inaba, S. 2011. Effects of tire inflation pressure and tractor velocity on dynamic wheel load and rear axle vibrations, Journal of Terramechanics 48(1): 3-16. http://doi.org/10.1016/j.jterra.2010.09.001

Pazooki, A.; Rakheja, S.; Cao, D. 2012. Modeling and validation of off-road vehicle ride dynamics, Mechanical Systems and Signal Processing 28: 679-695. http://doi.org/10.1016/j.ymssp.2011.11.006

Petković, D.; Issa, M.; Pavlović, N. D.; Zentner, L. 2015. Potential of adaptive neuro-fuzzy inference system for contact positions detection of sensing structure, Measurement 61: 234-242.

http://doi.org/10.1016/j.measurement.2014.10.040

Pérez, J.; Gajate, A.; Milanés, V.; Onieva, E.; Santos, M. 2010. Design and implementation of a neuro-fuzzy system for longitudinal control of autonomous vehicles, in 2010 IEEE International Conference on Fuzzy Systems (FUZZ), 18-23 July 2010, Barcelona, Spain, 1-6. http://doi.org/10.1109/FUZZY.2010.5584208

Rill, G. 2006. First order tire dynamics, in III European Conference on Computational Mechanics: Solids, Structures and Coupled Problems in Engineering: Book of Abstracts, 5-8 June, Lisbon, Portugal, 776-776. http://doi.org/10.1007/1-4020-5370-3_776 
Senatore, C.; Sandu, C. 2011. Off-road tire modeling and the multi-pass effect for vehicle dynamics simulation, Journal of Terramechanics 48(4): 265-276.

http://doi.org/10.1016/j.jterra.2011.06.006

Sokolovskij, E.; Prentkovskis, O.; Pečeliūnas, R.; KinderytèPoškienè, J. 2007. Investigation of automobile wheel impact on the road border, The Baltic Journal of Road and Bridge Engineering 2(3): 119-123.

Taghavifar, H. 2015. Artificial Neural Networks approach for prediction and modeling the coefficient of motion resistance, Journal of Advances in Vehicle Engineering 1(1): 6-13.

Taghavifar, H.; Mardani, A. 2016. Synthesis of the resultant force position on a radial ply tire of off-road vehicle with a comparative trend between some soft computing techniques, Neural Processing Letters 43(3): 627-639. http://doi.org/10.1007/s11063-015-9437-2

Taghavifar, H.; Mardani, A. 2014. On the modeling of energy efficiency indices of agricultural tractor driving wheels applying adaptive neuro-fuzzy inference system, Journal of Terramechanics 56: 37-47. http://doi.org/10.1016/j.jterra.2014.08.002

Taghavifar, H.; Mardani, A. 2013a. A knowledge-based Mamdani fuzzy logic prediction of the motion resistance coefficient in a soil bin facility for clay loam soil, Neural Computing and Applications 23(1): 293-302. http://doi.org/10.1007/s00521-013-1400-4

Taghavifar, H.; Mardani, A. 2013b. Investigating the effect of velocity, inflation pressure, and vertical load on rolling resistance of a radial ply tire, Journal of Terramechanics 50(2): 99-106. http://doi.org/10.1016/j.jterra.2013.01.005

Thomas, G.; Vantsevich, V. V. 2010. Wheel-terrain-obstacle interaction in vehicle mobility analysis, Vehicle System Dynamics: International Journal of Vehicle Mechanics and Mobility 48(S1): 139-156.

http://doi.org/10.1080/00423111003690496 